\title{
Mitochondrial solar sensitivity: evolutionary and biomedical implications
}

\author{
Andrei P. Sommer \\ Faculty of Science, ISRA University, Amman, Jordan \\ Correspondence to: Andrei P. Sommer. Visiting Professor, Faculty of Science, ISRA University, Amman, Jordan. \\ Email: andrei.sommer@alumni.uni-ulm.de; asommer@iu.edu.jo. \\ Provenance and Peer Review: This article was commissioned by the Editorial Office, Annals of Translational Medicine. The article did not undergo \\ external peer review. \\ Comment on: Sommer AP, Schemmer P, Pavláth AE, et al. Quantum Biology in Low Level Light Therapy: Death of a Dogma. Ann Transl Med 2019. \\ In press.
}

Submitted Nov 15, 2019. Accepted for publication Nov 29, 2019.

doi: 10.21037/atm.2019.11.100

View this article at: http://dx.doi.org/10.21037/atm.2019.11.100

Depending on cellular demand or oxidative stress, mitochondria produce adenosine triphosphate (ATP) or reactive oxygen species (ROS), thereby controlling the entire scale of cellular energy supply or disease, respectively. While deficiency in ROS can compromise the immune system, excessive ROS levels contribute to a large number of pathological conditions, including retinal, neurodegenerative and cardiovascular disease as well as cancer. Aging is also a process accelerated by ROS. There is one noninvasive tool allowing us to precisely control both mitochondrial ATP and ROS: red-to-near infrared (R-NIR) light. The understanding why and how R-NIR light interacts with mitochondria was missing so far in the literature. Here we present a unified model for the interaction of R-NIR photons with three mitochondrial key players involved in ATP and ROS generation: ATP synthase, cytochrome c (CYTc) and cytochrome c oxidase (COX). The new model allows us to predictably control ATP and ROS generation in mitochondria by R-NIR light. Furthermore, comparison of the action spectrum of R-NIR light related to mitochondrial ATP and ROS generation with the spectral solar irradiance on Earth, puts us in the position to propose an evolutionary model describing the coordinated interplay of solar irradiation and water on the development of mitochondria on Earth. It accurately predicts which wavelengths of light provides maximum benefit for any desired clinical application and provides valuable hints regarding a time point for the evolutionary provenance of the mitochondrion.

\section{Mitochondria: bacteria enslaved by cells}

The primary source of energy to the Earth is radiant energy from the Sun. After attenuation by the atmosphere, basically by scattering and absorption, the total solar irradiance reduces to the spectral solar irradiancethe normally incident solar spectrum at sea level on a clear day. Interestingly, the latter shows two pronounced minima in the R-NIR range: one around $750 \mathrm{~nm}$ and the other around $950 \mathrm{~nm}$ [figure 1 in ref (1)]. According to the literature, the cause for the first band is absorption by $\mathrm{H}_{2} \mathrm{O}$ and $\mathrm{O}_{2}$, while that of the second band is absorption by $\mathrm{H}_{2} \mathrm{O}$. Both $\mathrm{H}_{2} \mathrm{O}$ and $\mathrm{O}_{2}$ were present on primordial Earth. While abundance of $\mathrm{H}_{2} \mathrm{O}$ is traced back to the impact of comets carrying ice, that of $\mathrm{O}_{2}$ is attributed to the contribution of cyanobacteria. From this perspective it is plausible that primal mitochondria, supposed to originate from proteobacteria via enslavement and endosymbiosis, experienced minimal exposure to these wavelengths. Positively expressed: Primal mitochondria were protected from exposure to 750 and $950 \mathrm{~nm}$ light-as it is certainly true also today. Current theory on the origin of mitochondria (2) has been recently strongly challenged (3). Therefore, any piece of information which is applicable to a geological timescale and allows us to draw conclusions on the origin or evolution of mitochondria may help to come one step closer to the big picture regarding the evolution of Eukaryota on Earth. In this context, the finding that 750 and $950 \mathrm{~nm}$ light disrupts the electronic communication 
between CYTc and COX (4), complemented by the molecular picture describing the presence of 3 monolayers of water sandwiched between CYTc and COX (5), and our own results showing the perturbation of the molecular organization of 2-3 monolayers of interfacial water by R-NIR light $(6,7)$, thereby explaining the disruption of the electron flow between the two enzymes (8), becomes evolutionally challenging. As a result of the photonic perturbation, the space occupied by the interfacial water molecules will expand-a statistical process, corresponding to a drop in order. Notably, the intensity (irradiance) of the light emitting diodes (LED) used to disrupt the electron flow were on the order of the solar constant $\left(1,367 \mathrm{Wm}^{-2}\right)$. Since the primordial atmosphere of the Earth contained both $\mathrm{H}_{2} \mathrm{O}$ and $\mathrm{O}_{2}$, it seems far from speculative to assume that the disruption of the electron flow between CYTc and $\mathrm{COX}$ is a phenomenon caused by adaptation to the spectral solar irradiance-in which the wavelengths 750 and $950 \mathrm{~nm}$ were virtually absent or at least minimal. Since in nature mitochondria were never exposed to these two wavelengths, exposure to 750 and $950 \mathrm{~nm}$ artificial light could either result in an overreaction or no reaction, when compared to the rest of the R-NIR part of the solar spectrum. Interestingly, we see an overreaction as manifestation of a perturbation of the electron flow between CYTc and COX. The infrared regime of the solar spectrum shows further minima, however, as opposed to the R-NIR part of the spectrum, where the absorption by water is minimal, the absorption for water in the infrared part of the spectrum increases dramatically with the consequence that prolonged exposure of mitochondria to solar irradiation will increase the temperature of the internal aqueous content, a phenomenon different from the discrete perturbation of the nanoscopic interfacial water layers, where the action of R-NIR photons is restricted to bound water only, i.e., the nanoscopic interfacial water layers. In view of the temperature effect it is not clear if the absorption minima in the infrared regime of the solar spectrum will have the same effect as those in the R-NIR spectral regime.

If indeed mitochondria developed from intracellular enslavement of primitive bacteria, as it is believed today (3), then a simple method to isolate the potential mitochondrial ancestor, if not already extinct on the planet, consists in probing the response profile of the pool of potential bacterial candidates to their systematic irradiation with different wavelengths of laser or LED light. The species presenting the maximum similarity to the analogous response profile of mitochondria could, possibly, be an ancestor.

By extending the catalogue of the usual (epigenetic) factors by the parameter spectral sensitivity in the R-NIR regime this approach inspires new research in the field of evolutionary biology.

\section{Proteins: enslaved by nanoscopic interfacial water layers}

Previously we have put forward a model for the evolution of biological polymers such as RNA and DNA in which the process of polymerization was driven by the interplay of periodic diurnal light variations with nanoscopic interfacial water layers covering catalytic surfaces consisting of hydrogenated natural diamonds (9). This means, nanoscopic interfacial water layers are assumed to dictate the organization of biomolecules. Can we detect a similar natural interplay between light and nanoscopic interfacial water layers in mitochondria, and if so, what is its biological function? As it turns out in the following, the question is justified by observational evidence.

According to a unified model of protein dynamics (10), major internal protein motions are dictated by 2 monolayers of interfacial water surrounding the proteins. Protein surfaces in general, and enzyme surfaces in particular, are not rigid. Pockets and channels open and close (11). Individual function and interaction with proximal macromolecules is preceded by variations in both the viscosity and molecular order of the enslaving water mask (10).

It is well recognized that cells require a continuous supply of ATP to survive, and that the rate of ATP synthesis must be very precisely matched to that of ATP consumption. Most ATP synthesized in higher organisms is provided by mitochondrial oxidative phosphorylation, a metabolic pathway in which the energetically favorable reduction of oxygen to water is coupled to the energyrequiring synthesis of ATP. In other words, the function of oxidative phosphorylation is to synthesize ATP at the rate that it is being consumed via ion transport, mechanical work, molecular synthesis, etc. (12).

Apparently, cells should be protected from an overproduction of ATP triggered by the sun. Here, we mean an overdrive of the mitochondrial rotary motor via light-induced reduction of the viscosity of the nanoscopic interfacial water layers present within and around the mitochondrial rotary motor (13). Without further intervention, this process is likely to pull more electrons from 
CY'Tc to COX, eventually leading to a collapse of the entire respiratory system. To protect cells from such a catastrophe, the same light which is involved in reducing the viscosity of the nanoscopic interfacial water layers within and around the mitochondrial nano-turbine, thereby facilitating its normal rotation, seems to restrict the flow of electrons between CYTC and COX in a wavelength dependent manner (6). Here too, the target of the photons is the nanoscopic interfacial water layer present between CYTc and COX. This balancing reciprocal effect, safeguarding the survival of cells, is likely to occur in the same way in nature as it is observed in the laboratory: The terrestrial solar irradiance is sufficiently intense to induce both a drop in the viscosity of nanoscopic interfacial water layers defining the function of the mitochondrial rotary motor as well as to restrict the flow of electron between CYTc and COX. During experiments designed to explore the effect of R-NIR light on nanoscopic interfacial water layers in air and subaquatically (6), which we performed in dark, a colleague entered the laboratory and turned on the ceiling light. We noted that even this weak light had an effect on the nanoscopic interfacial water layers investigated by means of a quartz micro balance (unpublished results). Despite the negligible light intensity, when compared to that of the sun, there was a small but reproducible decrease in the number of water molecules forming the nanoscopic interfacial water layers attached to the quartz sensor. Interpreted in the light of the results of previous laboratory experiments, this result indicates that the terrestrial solar irradiance is capable to induce a volume expansion $(6,7)$ into the 3 interfacial monolayers of $\mathrm{H}_{2} \mathrm{O}$ between CYTc and COX, thus extending the length of the tunneling pathway between CYTc and COX. As elaborated in detail earlier (8), the probability for the transmission of electrons between the enzymes decreases exponentially with the width of the tunneling barrier in the mitochondrial photo fieldeffect transistor (photo-FET) with the gate represented by 3 monolayers of $\mathrm{H}_{2} \mathrm{O}$, the emitter by CYTc and the drain by $\mathrm{COX}$, where the transmission of the electrons through the gate is controlled by R-NIR photons.

\section{Spectral fingerprint of mitochondria}

The perfectly balanced functionality between the effect of R-NIR photons on the mitochondrial rotary motor and mitochondrial photo-FET points towards the theory that the two systems did not evolve separately but together. Presumably, it is neither easy to prove this hypothesis nor to fully reject it. What is certainly ascertained is that mitochondria respond differently to different wavelengths of light. Here, we focus exclusively on R-NIR light because of its relevance to biomedical applications, e.g., in low level light therapy (LLLT). The problem with LLLT is its inability to explain the observed and amply confirmed experimental and clinical results: According to the presently accepted model, the photon cell interaction mechanism relies on the absorption of the photons by mitochondria, specifically by the enzyme COX. As documented in our recent papers $(8,14)$, this hypothesis is rather ill-defined and not satisfactory and therefore it no longer can be counted as part of the theoretical framework proper. Instead, we have introduced two new schemes for the photon cell interaction: The first is inspired by nanotechnology and involves the application of the finding that R-NIR light reduces the viscosity of nanoscopic interfacial water layers, signifying its instrumentality to counteract the viscosity increase affecting the function of the mitochondrial rotary motor in oxidatively stressed cells, thereby instantly normalizing its rotational speed. As a result, the nanoturbine, which normally rotates with 9,000 rpm, increases its production of ATP. For oxidatively stressed cells, for instance, in an infected wound or the ischemic penumbra in a stroke, this means accelerated proliferation and cell survival, respectively. The second scheme comprises a balancing reciprocal effect and involves attenuation of the tunneling electron current between CYTc and COX in mitochondria by R-NIR photons, a generally valid quantum mechanical effect controlling the impact of R-NIR photons on mitochondria. Its general validity is derived from the discovery of the archetype of a photo-FET in mitochondria. Whereas the first scheme is dictated by observational evidence in laboratory experiments, the second follows from the results of Shimada et al. [Figure 5 in ref (5)] and 1:1 analogy with the intuitively simple model of the FET, first described by Lilienfeld in his patent (15). The impact of $\mathrm{R}-\mathrm{NIR}$ light on nanoscopic interfacial water layers similar to the $\mathrm{H}_{2} \mathrm{O}$ barrier (8) has been recently demonstrated in model experiments $(6,7,13)$.

\section{Quantum electronics in mitochondria- biomedical implications}

The biomedical implications of the two operational schemes is enormous, in particular with emphasis on LLLT. From an evolutionary point of view the coordinated interplay of the two schemes safeguards not only the propagation of simple Eukaryotes but also that of mammals where the 
intrinsic defense against ATP overproduction is usually controlled by the match between supply and demand fluxes. COX is not only responsible for the reduction of oxygen to water, but also for matching the rate of ATP synthesis to cellular demand for ATP (respiratory control) (12). However, when this equilibrium is disturbed, e.g., by exposure to high doses of R-NIR light or possibly during long exposure to strong sunlight, the second scheme comes into play as cellular protection. Moderately intense R-NIR light is known to be involved in the generation of ROS in mitochondria. Moreover, overproduction of ATP and ROS may contribute, e.g., to increased breast cancer risk (16). Hence, attenuation of the electron current between CYTc and COX appears as a natural regulatory mechanism to limit enhanced cell growth. Mitochondrial ROS can work as messengers in redox signaling, but can also damage both mitochondria and cells. The light usually administered during LLLT acts also as a double-edged sword: it simultaneously induces the production of mitochondrial ATP and ROS. Sustained exposure to ROS damages mitochondria, compromises the electron transport system and ultimately the mitochondrial DNA is damaged (17).

These aspects make the response of mitochondria to 750 and $950 \mathrm{~nm}$ light appear in a special light. Exposure of mitochondria to these wavelengths, applied at biostimulatory intensities, suppressed COX activity and prevented ROS generation-a new tool for normalizing hyperactive mitochondria (4). As envisaged by the authors, from the non-linear relationship between the mitochondrial membrane potential $\Delta \Psi \mathrm{m}$ and ROS, where ROS generation increases exponentially when the protonmotive force $(\Delta \Psi \mathrm{m})$ has reached a certain voltage threshold, even a small reduction of $\Delta \Psi \mathrm{m}$ through R-NIR-mediated inhibition of COX, would result in a large reduction of ROS. To neutralize an exponential process is only possible by a reciprocal exponential process. The aforementioned probability for the transmission of electrons from CYTc to COX, decreasing exponentially with the width of the tunneling barrier, implies that even a small increase in the barrier width will strongly inhibit or even totally block the flow of electrons, thereby perfectly counteracting the root cause of the generation of excess ROS induced by surpassing the $\Delta \Psi \mathrm{m}$ threshold. These reciprocal effects consistently explain the beneficial influence of 750 and $950 \mathrm{~nm}$ light on hyperactive mitochondria during conditions of massive oxidative stress, such as neuronal death during reperfusion injury following cerebral ischemia $(4,18)$. In general, ROS overproduction is detrimental to cells, and considered a key-factor for the development of several diseases, such as neurodegenerative diseases, cardiovascular disorders, and cancer (16). With the new understanding provided here, the targeted use of R-NIR light to prevent and limit ROS overproduction, where possible, should become a feasible alternative to pharmacological methods allowing us to put an end to the propagation of the vicious circle of ROS (17).

\section{The toolbox needed to uncover the evolution of mitochondria}

The Nobel Prize in Physics 1956 was awarded jointly to William Bradford Shockley, John Bardeen and Walter Houser Brattain "for their researches on semiconductors and their discovery of the transistor effect". Twenty years earlier Julius Edgar Lilienfeld filled the application for a patent entitled "method and apparatus for controlling electric currents". His invention first describing the principle of a field-effect transistor (FET) was patented four years later (15). Four years after the Nobel Prize for the transistor, Theodore Harold Maiman presented the ruby laser as the first functioning Laser (19). Another four years later the Nobel Prize in physics was awarded to Charles Hard Townes, Nicolay Gennadiyevich Basov and Aleksandr Mikhailovich Prokhorov "for fundamental work in the field of quantum electronics, which has led to the construction of oscillators and amplifiers based on the maser-laser principle.” In 1966 Endre Mester presented his first paper on the biomedical effect of non-thermal laser light, using a ruby laser (20). Written in Hungarian, his work was virtually invisible to the international scientific community. The general attitude towards the medical use of nonthermal laser light changed 1985 when Mester published a comprehensive presentation of the clinical results achieved by irradiation with R-NIR laser light delivered by ruby and He-Ne lasers (21). The catchy term Low Level Laser Therapy (LLLT) was coined and applications of the method began to grow. Today the biomedical use of lasers and LEDs experiences an exponential growth. With the upcoming of powerful LEDs operating in the R-NIR spectrum the nomenclature had to be adjusted accordingly: Today the acronym LLLT stands for the generalized term low level light therapy, where low indicates that the light intensities and doses used are non-thermal, i.e., biostimulatory.

In 1973 The Royal Swedish Academy of Sciences awarded the Nobel Prize in Physics to Leo Esaki, Ivar Giaever and Brian D Josephson "for their discoveries regarding tunneling phenomena in solids". While our understanding of the 
tunneling phenomenon in solids has largely contributed to the developments of semiconductor components, thereby triggering the entire development of the modern computer technology, quantum biology, the application of quantum mechanical concepts in biology, just starts to conquer the field of theoretical biology. However, in contrast to nanotechnology, biomedicine, in its current paradigm, has had wide success in applying classical models to living systems. In most cases, subtle quantum effects on (inter) molecular scales do not play a determining role in overall biological function (22). However, quantum mechanical tunneling, is one of the effects which helped to promote the reputation of quantum biology [ref (8) and references within].

In total three fundamental inventions in physics were the precondition to arrive to the understanding provided in the present editorial: laser, transistor and quantum tunneling. Apparently, two of the three principles were invented by nature. The elaborated insight puts us in the position to design a complete model of the interaction between photons and mitochondria, including practically the whole range of actors participating in the final episode of the mitochondrial respiration: ATP synthase, CYTc, COX, electrons, $\mathrm{H}_{2} \mathrm{O}$ and $\Delta \Psi \mathrm{m}$. The predictive capability of the proposed model is enormous. It exploits the complementary interplay of two powerful mechanisms in mitochondria: Reduction of the viscosity of nanoscopic interfacial water layers within and around the mitochondrial rotary motor and modulation of the electron current in the archetypical photo-FET. Both mechanisms are controlled by R-NIR light intensities comparable to that of the solar constant. The integral arrangement of the units forming the biological photo-FET is so unique that one would be surprised if nature had made no use of it. It does not only allow us to design better therapies using R-NIR light, but also, by taking into consideration the spectral solar irradiance, to obtain deeper insight into the evolution of mitochondria than it would be possible by utilization of existing evolutionary theories. Of special interest in this context are the pronounced minima in the R-NIR sector of the spectral solar irradiance at 750 and $950 \mathrm{~nm}$ (1), which perfectly coincide with the two LED wavelengths shown to inhibit the transfer of electrons between CYTc and COX. In concert with the small light intensities at the two minima of the spectrum and the circumstance that during the complete evolution mitochondria (assumed age $>1.45$ billion years) (23), were not exposed to intensive light except the sun, the simplest argument accounting for the effect of the LED wavelengths 750 and $950 \mathrm{~nm}$ is that the mitochondrial photo-FET apparatus (enzyme-nanoscopic interfacial water layer-enzyme) had no opportunity to adapt to the perturbation of the gate of the photo-FET (3 monolayers of $\mathrm{H}_{2} \mathrm{O}$ ), caused by these wavelengths. Since the strength of the perturbation is different for different wavelengths of light (6), and the nanoscopic interfacial water layer dictates protein reactions (10), it is clear that lack of adaptation of the coupled enzymes to the action of the wavelengths 750 and $950 \mathrm{~nm}$, e.g., lack of an adequate compensatory mechanism such as mechanical damping, will result in a stronger perturbation effect of the nanoscopic interfacial water layer confined between CYTc and COX, relative to other wavelengths of the R-NIR solar spectrum. Here, adaptation can be defined as cooperative survival response of the mitochondrial respiratory system to detrimental effects of the solar irradiation, e.g., its inhibitory effect on the tunneling of electrons across the $\mathrm{H}_{2} \mathrm{O}$ barrier confined between CYTc and COX (8). Without proper evolutionary adaptation to the spectral solar irradiance, operation of mitochondrial respiration would be presumably impossible and life would be restricted to a world without light.

\section{Acknowledgments}

Funding: None.

\section{Footnote}

Conflicts of Interest: APS serves as an unpaid editorial board member of ATM from Aug 2018 to Jul 2020.

Ethical Statement: The author is accountable for all aspects of the work in ensuring that questions related to the accuracy or integrity of any part of the work are appropriately investigated and resolved.

Open Access Statement: This is an Open Access article distributed in accordance with the Creative Commons Attribution-NonCommercial-NoDerivs 4.0 International License (CC BY-NC-ND 4.0), which permits the noncommercial replication and distribution of the article with the strict proviso that no changes or edits are made and the original work is properly cited (including links to both the formal publication through the relevant DOI and the license). See: https://creativecommons.org/licenses/by-nc-nd/4.0/.

\section{References}

1. Levinson R, Akbari H, Berdahl P. Measuring solar 
reflectance-Part I: Defining a metric that accurately predicts solar heat gain. Solar Energy 2010;84:1717-44.

2. Cavalier-Smith T. Origin of mitochondria by intracellular enslavement of a photosynthetic purple bacterium. Proc Biol Sci 2006;273:1943-52.

3. Martijn J, Vosseberg J, Guy L, et al. Deep mitochondrial origin outside the sampled alphaproteobacteria. Nature 2018;557:101-5.

4. Sanderson TH, Wider JM, Lee I, et al. Inhibitory modulation of cytochrome c oxidase activity with specific near-infrared light wavelengths attenuates brain schemia/ reperfusion injury. Sci Rep 2018;8:3481.

5. Shimada S, Shinzawa-Itoh K, Baba J, et al. Complex structure of cytochrome c-cytochrome c oxidase reveals a novel protein-protein interaction mode. EMBO J 2017;36:291-300.

6. Sommer AP, Zhu D, Försterling HD, et al. Crystalline Water at Room Temperature - Under Water and in Air. Cryst Growth Des 2008;8:2620-2.

7. Sommer AP, Hodeck KF, Zhu D, et al. Breathing volume into interfacial water with laser light. J Phys Chem Lett 2011;2:562-5.

8. Sommer AP, Schemmer P, Pavláth AE, et al. Quantum Biology in Low Level Light Therapy: Death of a Dogma. Ann Transl Med 2019. In press.

9. Sommer AP, Zhu D, Fecht HJ. Genesis on Diamonds. Cryst Growth Des 2008;8:2628-9.

10. Frauenfelder H, Chen G, Berendzen J, et al. A unified model of protein dynamics. Proc Natl Acad Sci U S A 2009; 106:5129-34.

11. Eyrisch S, Helms V. Transient pockets on protein surfaces involved in protein-protein interaction. J Med Chem 2007;50:3457-64.

12. Wilson DF, Harrison DK, Vinogradov A. Mitochondrial

Cite this article as: Sommer AP. Mitochondrial solar sensitivity: evolutionary and biomedical implications. Ann Transl Med 2020;8(5):161. doi: 10.21037/atm.2019.11.100 cytochrome c oxidase and control of energy metabolism: measurements in suspensions of isolated mitochondria. J Appl Physiol 2014;117:1424-30.

13. Sommer AP, Haddad MKh, Fecht HJ. Light effect on water viscosity: Implication for ATP biosynthesis. Sci Rep 2015;5:12029.

14. Sommer AP. Mitochondrial cytochrome c oxidase is not the primary acceptor for near infrared light-it is mitochondrial bound water: the principles of low-level light therapy. Ann Transl Med 2019;7:S13.

15. Lilienfeld JE. Method and apparatus for controlling electric currents. USPO, 1930, patent number 1,745,175.

16. Hecht F, Pessoa CF, Gentile LB, et al. The role of oxidative stress on breast cancer development and therapy. Tumour Biol 2016;37:4281-91.

17. Kowluru RA, Mishra M. Oxidative stress, mitochondrial damage and diabetic retinopathy. Biochim Biophys Acta 2015;1852:2474-83.

18. Strubakos CD, Malik M, Wider JM, et al. Non-invasive treatment with near-infrared light: A novel mechanismsbased strategy that evokes sustained reduction in brain injury after stroke. J Cereb Blood Flow Metab 2019:271678X19845149. [Epub ahead of print].

19. Maiman TH. Stimulated Optical Radiation in Ruby. Nature 1960;187:493-4.

20. Mester E. The use of the laser beam in therapy. Orv Hetil 1966;107:1012-6.

21. Mester E, Mester AF, Mester A. The biomedical effects of laser application. Lasers Surg Med 1985;5:31-9.

22. Marais A, Adams B, Ringsmuth AK, et al. The future of quantum biology. J R Soc Interface 2018. doi: 10.1098/ rsif.2018.0640.

23. Martin W, Mentel M. The Origin of Mitochondria. Nat Educ 2010;3:58. 